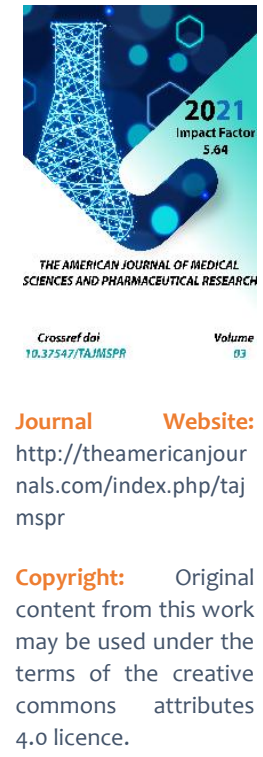

\title{
Morphological Verification Of Malignant Neoplasm Of The Urinary System With Multiple Bone Metastases
}

Shakhanova Shakhnoza Shavkatovna

Assistant Of Department Of Oncology And Radiology, Samarkand State Medical Institute, Samarkand, Uzbekistan

Nodir Mahammatkulovich Rakhimov

Associate Professor, Department Of Oncology And Radiology, Samarkand State Medical Institute, Samarkand, Uzbekistan

\section{ABSTRACT}

In metastatic renal cell carcinoma (mRCC), bone is the second most common site of metastases, occurring in one third of patients. Most bone metastases are found in the sacrum, pelvis, spine and proximal limbs. In addition, the majority of bone metastases are osteolytic with elements of destruction; mixed metastases also occur. This predisposes patients to skeletal events such as pathological fracture, spinal cord compression, which implies the prescription of radiation therapy or bone surgery.

\section{KEYWORDS}

Targeted therapy, bone metastasis of renal cell cancer.

\section{INTRODUCTION}

Key indicators for surveillance of morphological verification of urinary malignancy with multiple bone metastases. Skeletal events are associated with increased morbidity and have a very poor impact on patients' quality of life. In particular, bone pain is the most common type of pain caused by cancer, which may require opioid analgesics and palliative radiotherapy for pain. Prevention of SRE is therefore of paramount importance in this patient population [1-4]. Before the introduction of targeted therapy, these complications occurred $74-85 \%$ of the time. 


\section{MATERIALS AND METHODS}

We studied and evaluated the histological type of tumour, the level of malignancy, variants of bone metastases, complications of bone metastases, life expectancy from the stage of diagnosis to the initial response to treatment and to the case of skeletal complications in 129 patients [5-9].

The 1st group in 58 patients (prospective group, control group), who received specific antitumour therapy in combination with bisphosphonates (zolendronic acid), which is considered to be standard treatment for osteogenic metastases of solid tumours in Uzbekistan, was analyzed in the dynamics (before the treatment and in 3-6 months of therapy for 2 years) pain syndrome evaluation data (MDASI questionnaire, M. D. Anderson Symptom Inventory), instrumental examination (Rg-graphy, MSCT, RISK and MRI of bone metastases) and the level of bone resorption markers alkaline phosphotase, bone phosphotase and LDH;

The 2nd main group in 36 patients (prospective group) who received specific anti-tumour therapy in combination with targeted osteoprotective therapy with zoledronic acid supplemented with denosumab and radionuclide therapy (153samarium-oxabifor), Pain scores (BPI-SF, Brief Pain Inventory Short Form) and instrumental studies (X-rays, RBC and bone metastases MSC) were analysed over time (before treatment and every 3 months for 2 years);

Group 3 - 35 patients (prospective group) who received specific antitumour therapy in combination with targeted osteoprotective therapy, in whom zolendronic acid was not effective, were treated with denosumaboy and radionuclide therapy (153-samarium- oxabifor) Pain scores (BPI-SF, Brief Pain Inventory Short Form) and instrumental findings (X-ray, RIF and MSC of bone metastases) were analysed over time (before treatment and every 3 months for 2 years);

All patients had morphological (verified diagnosis of renal cell cancer (RCC), prostate cancer (PCC), urothelial bladder cancer (UBC) and testicular cancer (TNC) and bone metastases according to OSG or MSCT and/or MRI.

\section{RESULTS}

In the prospective group, the patients underwent a comprehensive clinical and laboratory examination, the aim of which was to clarify the extent of the disease as well as to assess the functional indices of the internal organs [12-16]. After routine examination also with obligatory determination of total alkaline phosphatase (ALP), acid phosphatase (AP), lactate dehydrogenase (LDH) and serum calcium levels. The following imaging techniques were used to determine the sites of bone lesions, as well as the structure of the lesions and the number of affected bones:

- Radioisotope scans were performed in 2 projections in whole-body imaging mode. The radiopharmaceutical 153-samarium oxabifor was used as a radioisotope indicator. $153 \mathrm{Sm}$ OXABIFOR was injected intravenously 60-75 $\mathrm{mCi}-3.0 \mathrm{ml}$ (at the rate of $0.5-1.5$ megacuries $(\mathrm{mCi}) / \mathrm{kg}, \quad 1 \mathrm{mCi}=37$ megabecuries $(\mathrm{MBq})$ depending on patient body weight) in a dose of $1 \mathrm{mCi}$ (37 $\mathrm{MBq}$ ) per $1 \mathrm{~kg}$ weight intravenously through an angiotape followed by infusion of $400 \mathrm{ml}$ of physiological solution. Images were taken with a high-resolution lowenergy collimator at a table speed of 12-16 $\mathrm{cm} / \mathrm{min}$. The scanning procedure took 15-20 min. Post-therapy efficacy was assessed at follow-up observations at 1 and 3 months after 
the administration of $153 \mathrm{Sm}$-OXABIFOR. The distribution and accumulation of Samaria-153 oxabifor was studied by performing wholebody scans on a Mediso 89 dual-detector gamma camera immediately after administration of the RFP, 6 and 24 hours later. After administration of Samaria-153 oxabiphor, gamma ray emission levels were measured in the room at a distance of 1 meter from the patient. The patients were kept in the ward until the level of gamma-quanta radiation at the distance of 1 metre from the patient's heel did not exceed $3 \mu \mathrm{Sv}$ per hour. This level was determined using dosimeterradiometer IRD-02B1 and was measured from the moment of drug administration, after 6 and 24 hours. This phase of the study was performed at the Department of Interventional Radiology of the Vokhidov Republican Specialized Scientific-Practical Center of Surgery. Vohidov). In the image analysis the area of interest (metastasis) was compared with a symmetrical zone in the contralateral skeleton area and the relative degree of RAF accumulation was determined throughout the study. The images were interpreted as follows: decrease in RFP inclusion rate - positive dynamics, increase in area and intensity of RFP accumulation negative dynamics, no dynamics - stabilization;

Radionuclide preparation $153-\mathrm{Sm}$ oxabifor is a colourless, transparent solution containing 240-740 MBq samarium-153 in $1 \mathrm{ml}$; Radionuclide and radiochemical purity is at least $90 \%, \mathrm{pH}$ 5.0-7.0. The preparation is sterile and apirogenic. 153-samarium emits u-quanta with energies of $69.7 \mathrm{keV}$ and $103 \mathrm{keV}$ with yields of $5.4 \%$ and $28 \%$ respectively and betaradiation energy of $203 \mathrm{keV}, 229$ and $268 \mathrm{keV}$ with yields of $35 \%, 43 \%$ and $21 \%$ respectively. The half-life of $153 \mathrm{Sm}$ is 46.2 hours. After intravenous injection the radionuclide accumulates predominantly in bone metastases. Such accumulation is caused by the chemical tropism of the transport compound (oxabiphor) to the zones of altered hypermetabolism (metastases, fractures and active sites of inflammation). Once in the metastatic nidus, the samarium exposes it to local radiation, mainly by bettaradiation. One month after the introduction of 153 Sm-OXABIFOR OB changed as follows: 21 $(60 \%)$ patients had a decrease of pain, 6 (18.7\%) an increase of pain, 5 (15.6\%) had no effect. Three months after administration of 153 Sm-OXABIFOR

OB was as follows: $2(6.3 \%)$ stopped pain, $27(84.3 \%)$ reduced pain, $2(6.3 \%)$ increased pain. Bone palliative effect obtained after 1 and 3 months: complete pain relief in 3(10\%) and $2(6.3 \%)$ patients, marked pain relief in $11(34 \%)$ and $10(31 \%)$ patients Mild pain relief in $10(31 \%)$ and $11(34 \%)$ patients and no effect in $8(25 \%)$ and $9(28 \%)$ patients respectively. Patient mobility. Pain relief was accompanied by improvement in patient mobility. The mean values and changes in quality of life (QoL) on the basis of the Karnofsky Scale (KS) were as follows: analgesic discontinuation $15.6 \%$ and $21.9 \%$, pain relief with NSAIDs $31.3 \%$ and $37.5 \%$, mild opiates $31.3 \%$ and $28.1 \%$, strong opiates $21.9 \%$ and $25 \%$ after 1 and 3 months respectively. As can be seen from the table, when an integrated approach is prescribed in the treatment of osteogenic metastasis of prostate cancer, there is a tendency for a decrease in the intake of analgesics. Pain relief was accompanied by an improvement in the mobility of the patient. Mean values and changes in quality of life (QOL) $n$ Most patients had grade 1 or 2 infections and did not bleed for 3 months after treatment with 153 Sm-OXABIFOR. Haematological toxicity before and at 1 and 3 months after 153 SmOXABIFOR is summarised in the table. SmOXABIFOR therapy for bone pain relief in prostate cancer patients was effective, safe and well tolerated. The analgesic effect with a 
simultaneous improvement of the patient's mobility and with a reduction in the required dosage of analgesics. Haematological toxicity after administration of Sm-OXABIFOR was moderate and transient. and based on the Karnofsky Scale (KS) the whole body of a patient with metastatic prostate cancer shows several osteoblastic lesions of the axial and appendicular skeleton (arrows). Images were obtained 4 hours after administration of a therapeutic dose (70 mCi) of Sm-153-oxabifor. Areas of osteoblasts are perfectly matched.

\section{CONCLUSIONS}

1. Denosumab, a monoclonal antibody from the RANKL antagonist group, reduces the incidence of skeletal complications ( 0.33 vs. 0.55 skeletal complications per patient per year when compared to Zoledronic acid, $\mathrm{p}=0.13$ ) and pain syndrome (by $66 \%$ and $35 \%$ after 12 months of use, respectively 24 .

2. We developed a comprehensive impact on all pathogenetic mechanisms of bone metastasis and a strategy for the clinical study of drugs that affect bone metabolism. The use of radiopharmotherapy, targeted therapy reduces the use of narcotic analgesics, reduces hypercalcemia. Our proposed therapeutic complex using bisphosphonates, monoclonal antibodies and radionuclide therapy show their effectiveness in periods of disease progression, which has the greatest practical value, which demonstrates new opportunities in the effective treatment of cancer patients in this category.

\section{REFERENCES}

1. Ahmadzadehfar, H., Essler, M., Rahbar, K., \& Afshar-Oromieh, A. (2018). Radionuclide therapy for bone metastases: utility of scintigraphy and PET imaging for treatment planning. PET clinics, 13(4), 491-503.

2. Alavi, M., Khajeh-Rahimi, F., Yousefnia, H., Mohammadianpanah, M., Zolghadri, S., Bahrami-Samani, A., \& Ghannadi-Maragheh, M. (2019). 177Lu/153Sm-Ethylenediamine tetramethylene phosphonic acid cocktail: A novel palliative treatment for patients with bone metastases. Cancer biotherapy \& radiopharmaceuticals, 34(5), 280-287.

3. Alsharef, S., Alanazi, M., Alharthi, F., Qandil, D., \& Qushawy, M. (2020). Review about radiopharmaceuticals: preparation, radioactivity, and applications. International Journal of Applied Pharmaceutics, 8-15.

4. $\quad$ Casadei, R., Drago, G., Di Pressa, F., \& Donati, D. (2018). Humeral metastasis of renal cancer: Surgical options and review of literature. Orthopaedics \& Traumatology: Surgery \& Research, 104(4), 533-538.

5. Dash, A., Das, T., \& Knapp, F. F. R. (2020). Targeted radionuclide therapy of painful bone metastases: past developments, current status, recent advances and future directions. Current medicinal chemistry, 27(19), 3187-3249.

6. Dolezal, J., Vizda, J., \& Odrazka, K. (2007). Prospective evaluation of samarium-153-EDTMP radionuclide treatment for bone metastases in patients with hormone-refractory prostate cancer. Urologia internationalis, 78(1), 50-57.

7. Wong, E. C., \& Kapoor, A. (2020). Does Bone-targeted Therapy Benefit Patients with Metastatic Renal Cell Carcinoma?. Translational oncology, 13(2), 241-244. 
8. $\quad$ Erfani, M., Rahmani, N., Doroudi, A., \& Shafiei, M. (2017). Preparation and evaluation of rhenium-188pamidronate as a palliative treatment in bone metastasis. Nuclear medicine and biology, 49, 1-7.

9. Fallon, M., Giusti, R., Aielli, F., Hoskin, P., Rolke, R., Sharma, M., \& Ripamonti, C. I. (2018). Management of cancer pain in adult patients: ESMO Clinical Practice Guidelines. Annals of Oncology, 29, iv166-iv191.

10. Shomurodov. K.E. (2010). Features of cytokine balance in gingival fluid at odontogenicphlegmon of maxillofacial area. Doctor-aspirant. 42(5.1). pp.187192.

11. Tillyashaykhov M. N., Rakhimov N. M. Khasanov Sh. T. (2019). Features of Clinical Manifestation of the bladder cancer in young people. Doctor Bulletin. Samarkand. №2. pp. 108-113.

12. Ilkhomovna, K. M., Eriyigitovich, I. S., \& Kadyrovich, K. N. (2020). Morphological Features of Microvascular Tissue of The Brain At Hemorrhagic Stroke. The American Journal of Medical Sciences and Pharmaceutical Research, 2(10), 53-59.

13. Ziyadullaev, S., Elmamatov, O., Raximov, N., \& Raufov, F. (2020). Cytogenetic and immunological alterations of recurrent bladder cancer. European Journal of Molecular \& Clinical Medicine, 7(2), 1877-1883.

14. Tillyashaykhov M, Rakhimov N, Boyko E, Ravshan Kh. (2020). Comparative characteristics of en-block resection and conventional transurethral resection in nonmuscular invasive bladder cancer (metaanalysis). Journal of Biomedicine and Practice, 6(5), pp.124-131.

15. Rahimov M. Nodir, Shakhanova Sh. Shaxnoza, Raupov Farhod. (2020).
Development of new approaches in treatment of metastatic renal cell carcinomall Journal of research in health science. 5(4). pp.82-95.

16. Saidkulov, B., Abduraxmonov, A., \& Rahimov, N. (2020). Recurrent ovarian cancer: mechanisms of development of peritoneal malignant ascites. European Journal of Molecular \& Clinical Medicine ISSN, 2515-8260. 